\title{
ON SUPERCOMPLETE UNIFORM SPACES
}

\begin{abstract}
AARNO HOHTI
ABSTRACT. We show that a uniform space $\mu X$ is supercomplete if, and only if, the Ginsburg-Isbell locally fine coreflection of $\mu X \times \mathscr{F} \beta X$ is equinormal.
\end{abstract}

1. Introduction. J. Isbell calls a uniform space $\mu X$ supercomplete if the uniform hyperspace $H(\mu X)$ of closed subsets of $X$ is complete. He proved in [4] that $\mu X$ is supercomplete if, and only if, $X$ is paracompact and $\lambda \mu=\mathscr{F}_{X}$, where $\mathscr{F}_{X}$ is the fine uniformity of $X$. (On the other hand, Morita proved in [7] that the uniform hyperspace $K(\mu X)$ of all compact subsets of $X$ is complete if and only if $\mu X$ is complete.) Tamano established in 1960 (see [9]) the following famous result: a completely regular space $X$ is paracompact whenever $X \times \beta X$ is normal. It seems reasonable to ask if it is possible to give a Tamano-like "finitary" characterization of supercomplete uniform spaces by means of their Čech-Stone compactifications. First of all, we will recall some familiar definitions. A quasiuniformity is a filter of coverings ordered by the relation of refinement. If $\mu$ and $\nu$ are quasiuniformities, then $\mu / \nu$ is the quasiuniformity consisting of all covers $\left\{V_{i} \cap W_{j}^{i}\right\}$, where $\left\{V_{i}\right\}_{i} \in \nu$ and for each $i,\left\{W_{j}^{i}\right\}_{j} \in \mu$. If $\mu$ is a quasiuniformity, let $\mu^{(0)}=\mu$. If $\mu^{(\alpha)}$ is defined for all $\alpha<\beta$ and $\beta$ is a limit ordinal, let $\mu^{(\beta)}=\bigcup\left\{\mu^{(\alpha)}: \alpha<\beta\right\}$. On the other hand, if $\beta=\alpha+1$, then we define $\mu^{(\beta)}=\mu^{(\alpha)} / \mu^{(\alpha)}$. There is an ordinal $\alpha$ such that $\mu^{(\alpha)}=$ $\mu^{(\alpha+1)}$ and for which we define $\lambda \mu=\mu^{(\alpha)}$. In case $\mu$ is a uniformity, it can be shown that [1, Theorem 4.4] $\lambda \mu$ is the coarsest locally fine [1] uniformity finer than $\mu$. The operator $\lambda$ is called the Ginsburg-Isbell locally fine coreflection. The precompact reflection of $\mu$ will be denoted by $p \mu$. A uniform space is equinormal if every binary open cover of the space is uniform. The symbol $\mathscr{T} \mid A$ (resp. $\mu \mid A$ ) denotes the trace of the family $\mathcal{T}$ on $A$ (resp. the induced quasiuniformity of $A$ ).

2. The result. Tamano proved the following lemma in [9].

LEMMA 1. A completely regular space $X$ is paracompact if and only if for each compact $K \subset \beta X \sim X$ there is a locally finite open cover $\mathcal{T}$ of $X$ such that $\operatorname{cl}_{\beta X} V \cap K$ $=\varnothing$ for all $V \in \mathcal{T}$.

We will extend Lemma 1 to " $\lambda$-uniform" covers.

Received by the editors February 1, 1982 and, in revised form, June 8. 1982. The contents of this paper were presented to the 10th Winter School in Sumava, Czechoslovakia, in February 1982 when the author visited the Czechoslovak Academy of Sciences under an exchange programme between CSAV and the Academy of Finland.

1980 Mathematics Subject Classification. Primary 54E15, 54B20: Secondary 54D18. 
LEMMA 2. A uniform space $\mu X$ is supercomplete if and only if for each compact $K \subset \beta X \sim X$ there is a $\mathfrak{V} \in \lambda \mu$ such that $\operatorname{cl}_{\beta X} V \cap K=\varnothing$ for all $V \in \mathcal{V}$.

Proof. To prove sufficiency, suppose that the condition given in Lemma 2 holds. By Lemma $1, X$ is paracompact since each element of $\lambda \mu$ has a locally finite open refinement $[5,1.14]$. We will show that $\lambda \mu=\widetilde{F}_{X}$. To this end, let $\mathcal{W}$ be an open cover of $X$. For each element $V$ of $\mathcal{T}$ choose an open set $V^{*}$ in $\beta X$ with $V=V^{*} \cap X$. Then $K=\beta X \sim \cup\left\{V^{*}: V \in \mathcal{V}\right\}$ is compact. Hence, there exists a $\mathscr{U} \in \lambda \mu$ such that $\mathrm{cl}_{\beta X} W \cap K=\varnothing$ for all $W \in \mathcal{O} \int$. It is not difficult to see that $\mathscr{O} f$ refines the cover $\chi^{<\omega}$ consisting of all finite unions of sets from $\mathcal{T}$. As $\mathcal{T}$ is arbitrary, Theorems 2 and 3 in [8] show that $\widetilde{F}_{X}=p \lambda \mu / \lambda \mu$. But $p \lambda \mu / \lambda \mu=\lambda \mu$. Hence $\mu X$ is supercomplete.

On the other hand, let $\mu X$ be supercomplete and assume that $K \subset \beta X \sim X$ is compact. By Isbell's result $X$ is paracompact and $\lambda \mu={\widetilde{{ }_{F}}}_{X}$. Let $\mathcal{T}_{\text {be }}$ as in Lemma 1. Since $X$ is paracompact, we have $\mathcal{T} \in \mathscr{F}_{X}=\lambda \mu$, as desired.

To prove the main result, we shall need an auxiliary derivative introduced in [3]. Let $\mu_{0}^{(0)}=\mu$. If $\mu_{0}^{(\alpha)}$ is defined for all $\alpha<\beta$ and $\beta$ is a limit ordinal, let $\mu_{0}^{(\beta)}=$ $\cup\left\{\mu_{0}^{(\alpha)}: \alpha<\beta\right\}$; if $\beta=\alpha+1$, then we define $\mu_{0}^{(\beta)}=\mu_{0}^{(\alpha)} / \mu$. The following basic lemma can be found in [3]. The statement is proved by a transfinite induction argument.

LEMMA 3. For each $\alpha$ there is a $\sigma(\alpha)$ such that $\mu^{(\alpha)} \subset \mu_{0}^{(\sigma(\alpha))}$.

It follows that there is an ordinal $\alpha$ with $\mu_{0}^{(\alpha)}=\lambda \mu$.

Finally, we shall need the following result proved in [2].

Lemma 4. Let $\mu X$ be a uniform space and let $K$ be a compact space. Then $\left(\bar{T}_{X} \times \bar{T}_{K}\right)^{(1)}=\widetilde{T}_{(X \times K)}$.

Note that in the preceding lemma both $\left(\bar{F}_{X} \times \overline{\mathscr{F}}_{K}\right)^{(1)}$ and $\bar{y}_{(X \times K)}$ are uniformities on $X \times K$.

THEORFM. A uniform space $\mu X$ is supercomplete if, and only if, the Ginsburg-Isbell locally fine coreflection of $\mu X \times \widetilde{Y} \beta X$ is equinormal.

Proof. To prove sufficiency, we will proceed by transfinite induction. Let $K \subset \beta X \sim X$ be compact. Then $X \times K$ and $\Delta(X)$ (the diagonal of $X \times X)$ are closed and disjoint subsets of $X \times \beta X$. Define $G_{1}=(X \times \beta X) \sim(X \times K)$ and $G_{2}=(X \times \beta X) \sim \Delta(X)$. Then $\hat{\xi}=\left\{G_{1}, G_{2}\right\}$ is a binary open cover of $X \times \beta X$. Let $P(\alpha)$ be the following statement: if $\nu Y$ is a uniform space, $K \subset \beta Y \sim Y$ is compact, is defined as above, and $\bar{\varphi} \in\left(\nu \times \bar{T}_{\beta Y}\right)_{0}^{(\alpha)}$, then there is a $\widetilde{T} \in \nu_{0}^{(\alpha)}$ such that $\mathrm{cl}_{\beta Y} V \cap K=\varnothing$ for all $V \in \Upsilon$. Theorem 5.1.2 in [2] shows that $P(\alpha)$ is valid for $\alpha=1$. For the induction hypothesis, suppose that $P(\alpha)$ holds whenever $1 \leqslant \alpha<\tau$. Let $\mathcal{G} \in\left(\mu \times \mathscr{F}_{\beta X}\right)_{0}^{(\tau)}$. If $\tau$ is a limit ordinal, then the induction hypothesis implies directly that there is a $\mathcal{T} \in \mu_{0}^{(\tau)}$ with the desired property. Thus, we can assume that $\tau=\alpha+1$. There is a uniform cover \& of $X \times \beta X$ such that for each $U \in$ ol we have $\mathfrak{i}_{\mid} \mid U \in\left[\left(\mu \times \widetilde{F}_{\beta X}\right) \mid U\right]_{0}^{(\alpha)}$. Choose $\left\{U_{i}\right\} \in \mu$ and $\{V\} \in \widetilde{F}_{\beta X}$ such that $\left\{U_{i} \times\right.$ $\left.V_{j}\right\}<$ थl. As $\beta X$ is compact we can assume that $\left\{V_{j}\right\}=\left\{V_{1}, \ldots, V_{n}\right\}$. We can assume 
that each $U_{i}$ is closed. It follows from the hypothesis of the theorem that $X$ is normal. By Urysohn's extension theorem, $U_{i}$ is $C^{*}$-embedded in $X$ and consequently $\beta\left(U_{i}\right)$ is homeomorphic (and thus unimorphic) to $\mathrm{cl}_{\beta X} U_{i}$. For this reason, we can write $\beta\left(U_{i}\right)=\operatorname{cl}_{\beta X} U_{i}$. Now $\mathcal{G} \mid\left(U_{i} \times V_{j}\right) \in\left[\left(\mu \times \bar{y}_{\beta X}\right) \mid\left(U_{i} \times V_{j}\right)\right]_{0}^{(\alpha)}$ for all $i$ and $j$. As $\left(\mu \times \bar{Y}_{\beta X}\right)_{0}^{(\alpha)}$ is a quasiuniformity it is easy to see that for each $i$, the restriction of $i$ to $U_{1} \times \beta X$ belongs to $\left[\left(\mu \times \widetilde{F}_{\beta X}\right) \mid\left(U_{i} \times \beta X\right)\right]_{0}^{(\alpha)}$; hence $\mathcal{Q}_{\mid} \mid\left(U_{1} \times \beta\left(U_{i}\right)\right)$ belongs to $\left[\left(\mu \times \overline{\mathscr{Y}}_{\beta X}\right) \mid\left(U_{1} \times \beta\left(U_{1}\right)\right)\right]_{0}^{(\alpha)}$. Further, note that

$$
G_{1} \cap\left(U_{1} \times \beta\left(U_{1}\right)\right)=\left(U_{1} \times \beta\left(U_{1}\right)\right) \sim(X \times K)=\left(U_{1} \times \beta\left(U_{1}\right)\right) \sim\left(U_{1} \times K_{1}\right)
$$

where $K_{t}=K \cap \beta\left(U_{1}\right)$ and

$$
G_{2} \cap\left(U_{1} \times \beta\left(U_{1}\right)\right)=\left(U_{1} \times \beta\left(U_{1}\right)\right) \sim \Delta\left(U_{1}\right) .
$$

Finally, note that $\left[\left(\mu \times \bar{x}_{\beta X}\right) \mid\left(U_{1} \times \beta\left(U_{1}\right)\right)\right]_{()}^{(\alpha)}$ is the same quasiuniformity as $\left(\mu \mid U_{1} \times \bar{s}_{\beta\left(C_{,}\right)}\right)_{0}^{(\alpha)}$. Therefore, the induction hypothesis applies and we can find a cover $\left\{W_{h}^{\prime}\right\}_{h}$ in $\left(\mu \mid U_{i}\right)_{0}^{(\alpha)}$ such that $\mathrm{cl}_{\beta\left(C_{i}\right)} W_{h}^{\prime} \subset \beta\left(U_{i}\right) \sim K_{i}$ for all $k$. Now

$$
\mathrm{cl}_{\beta\left(C_{i}\right)} W_{h}^{i}=\mathrm{cl}_{\mathrm{cl}_{\beta,}, C_{i}} W_{h}^{i}=\mathrm{cl}_{\beta X} W_{h}^{\prime} \cap \mathrm{cl}_{\beta, X} U_{l} \text {. }
$$

Choose a closed cover $\left\{\tilde{W}_{l}^{\prime}\right\}_{l} \in \mu_{0}^{(\alpha)}$ such that $\left\{U_{i} \cap \tilde{W}_{l}^{\prime}\right\}<\left\{W_{h}^{\prime}\right\}$. Then $\left\{U_{1} \cap \tilde{W}_{l}^{\prime}\right\}_{\text {I.l }}$ $\in \mu_{0}^{(\alpha)} / \mu=\mu_{0}^{(\alpha+1)}\left(\right.$ since $\left.\left\{U_{i}\right\} \in \mu\right)$ and

$$
\begin{aligned}
\operatorname{cl}_{\beta X}\left(U_{l} \cap \tilde{W}_{l}^{\prime}\right) & =\mathrm{cl}_{\beta X} U_{i} \cap \mathrm{cl}_{\beta X} \tilde{W}_{l}^{\prime} \subset \mathrm{cl}_{\beta X} U_{l} \cap \mathrm{cl}_{\beta X} W_{h(1)}^{\prime} \\
& =\mathrm{cl}_{\beta\left(U_{1}\right)} W_{h(1)}^{\prime} \subset \beta\left(U_{i}\right) \sim K \subset \beta X \sim K
\end{aligned}
$$

since $X$ is normal (see, for example, [6, p. 764]). Hence $P(\tau)$ is valid. This completes the induction. Since $\lambda\left(\mu X \times \bar{Y}_{\gamma} \beta\right)$ is equinormal, we have $s \in \lambda\left(\mu \times \bar{r}_{\beta X}\right)$. As $P(\alpha)$ is valid for all $\alpha$, it follows from Lemma 3 that there is a $\breve{Y} \in \lambda \mu$ such that $\mathrm{cl}_{\beta X} V \cap K=\varnothing$ for all $V \in T$. By Lemma 2, $\mu X$ is supercomplete.

Necessity follows directly from Exercise VII.8.c) in [5]: the product of a supercomplete space and a compact space is supercomplete. We give a proof for the reader's convenience. Let $\mu X$ be supercomplete and let $K$ be compact. Then $X$ is paracompact and $\lambda \mu X=\bar{y} X$. Hence $X \times K$ is paracompact. By Lemma 4 , the

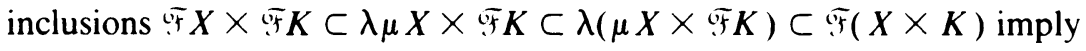

$$
\bar{r}_{(X \times K)}=\left(\bar{F}_{X} \times \overline{\mathscr{T}}_{K}\right)^{(1)} \subset\left(\lambda\left(\mu \times \bar{T}_{K}\right)\right)^{(1)}=\lambda\left(\mu \times \bar{r}_{K}\right) \subset \bar{r}_{(X \times K)}
$$

and thus $\lambda\left(\mu \times \bar{Y}_{K}\right)=\bar{v}_{(X \times K)}$. Hence, the proof is complete.

ACKNOWLEDGEMENT. The author acknowledges the inspiration given by Jan Pelant during the Fifth Prague Topological Symposium. The research was partially supported by a grant from the Academy of Finland.

\section{REFERENCES}

1. S. Ginsburg and J. R. Isbell, Some operators on uniform spaces. Trans. Amer. Math. Soc. 93 (1959). $145-168$.

2. A. Hohti. On uniform paracompactness, Ann. Acad. Sci. Fenn. Ser. A I Math. Dissertationes 36 (1981).

3. On the (iinshurg-Ishell dericatices and ranks of metric spaces (preprint) 
4. J. R. Isbell, Supercomplete spaces, Pacific J. Math. 12 (1962), 287-290.

5. U_ Uniform spaces, Math. Surveys, no. 12, Amer. Math. Soc., Providence, R. I.. 1964.

6. J. Keesling. Normality and properties related to compactness in hyperspaces, Proc. Amer. Math. Soc. 24 (1970), 760-766

7. K. Morita. Completion of hyperspaces of compact subsets and topological completion of open-closed maps. (jeneral Topology and Appl. 4 (1974), 217-33.

8. M. D. Rice, A note on uniform paracompactness, Proc. Amer. Math. Soc. 62 (1977), 359-362.

9. H. Tamano, On paracompactness. Pacific J. Math. 10 (1960). 1043-1047.

University of Helsinki, Hallituskatu 15, SF-00100 Helsinki 10, Finland 